The Astronomical Journal, 116:801-812, 1998 August

(C) 1998. The American Astronomical Society. All rights reserved. Printed in U.S.A.

\title{
A PHOTOMETRIC AND SPECTROSCOPIC STUDY OF THE SOUTHERN OPEN CLUSTERS PISMIS 18, PISMIS 19, NGC 6005, AND NGC 6253
}

\author{
ANDrÉs E. Piatti ${ }^{1,2}$ AND JuAN J. Clariá ${ }^{2}$ \\ Observatorio Astronómico, Universidad Nacional de Córdoba, Laprida 854, 5000 Córdoba, Argentina; andres@oac.uncor.edu, claria@oac.uncor.edu \\ EDUARDO BICA ${ }^{2}$ \\ Departamento de Astronomia, Universidade Federal do Rio Grande do Sul, C.P. 15051, Porto Alegre, 91500-970, RS, Brazil; bica@if.ufrgs.br \\ DOUG GeISLER \\ Kitt Peak National Observatory, National Optical Astronomy Observatories, P.O. Box 26732, Tucson, AZ 85726; doug@noao.edu \\ AND \\ DANTE MINNITI \\ Lawrence Livermore National Laboratory, L-413, P.O. Box 808, Livermore, CA 94550; dminniti@igpp.llnl.gov \\ Received 1997 October 3 ; revised 1998 April 24
}

\begin{abstract}
CCD observations in the $B, V$, and $I$ passbands have been used to generate color-magnitude diagrams (CMDs) for the southern open cluster candidates Pismis 18, Pismis 19, and NGC 6005, as well as for the old open cluster NGC 6253. The sample consists of about 1550 stars reaching down to $V \sim 19$ mag. From analysis of the CMDs, the physical reality of the three cluster candidates is confirmed and their reddening, distance, and age are derived, as well as those of NGC 6253. In addition, integrated spectra for Pismis 18, Pismis 19, and NGC 6253 covering a range from 3500 to $9200 \AA$ were obtained. The reddening, age, and metallicity of these three clusters were derived from Balmer and Ca II triplet equivalent widths by comparing the observed spectra with those of template clusters. The photometric and spectroscopic results allow us to conclude that Pismis 18, Pismis 19, and NGC 6005 are intermediate-age $(t \approx 1 \mathrm{Gyr})$, genuine open clusters, while NGC 6253 has an age $t \approx 5 \mathrm{Gyr}$. All these clusters are located approximately toward the Galactic center, between 1.5 and $2.7 \mathrm{kpc}$ from the Sun. Their metallicities range over values that are typical of moderately metal-poor to moderately metal-rich open clusters.
\end{abstract}

Key words: galaxies: star clusters - open clusters and associations: individual (Pismis 18, Pismis 19, NGC 6005, NGC 6253)

\section{INTRODUCTION}

Galactic open clusters have long been used to study the structure and evolution of the disk of our own Galaxy, as well as to improve our knowledge about the formation, structure, and evolution of the stars. This is essentially due to the relative ease of determining their reddenings, distances, and metallicities with considerable precision (see, e.g., Janes, Tilley, \& Lyngå 1988; Friel \& Janes 1993; Piatti, Clariá, \& Abadi 1995). In spite of the uncertainties in dating them, they also provide more accurate ages than do any other disk objects. Moreover, a database of open clusters with a wide age range is presently available. The latter covers an important fraction of the timescale over which the evolution of the Galactic disk has occurred.

In particular, open clusters located toward the Galactic center play an important role as they offer the possibility to trace the structure and evolution of the inner disk more precisely. Many of these objects, however, have not been studied in detail yet because they are affected by high interstellar absorption and/or strong field star contamination (Piatti, Bica, \& Clariá 1998). This is the case of the open cluster candidates Pismis 18 (B1950.0 coordinates

\footnotetext{
${ }^{1}$ Visiting Astronomer, University of Toronto (David Dunlap Observatory) 24 inch telescope, Las Campanas, Chile.

${ }^{2}$ Visiting Astronomer, Complejo Astronómico El Leoncito, which is operated under agreement between the Consejo Nacional de Investigaciones Científicas y Técnicas de la República Argentina and the National Universities of La Plata, Córdoba, and San Juan, Argentina.
}

$\left.\alpha=13^{\mathrm{h}} 33^{\mathrm{m}} 07^{\mathrm{s}}, \quad \delta=-61^{\circ} 57^{\prime} 30^{\prime \prime} ; \quad l=308^{\circ} 16, \quad b=+0.2\right)$, Pismis $19\left(\alpha=14^{\mathrm{h}} 26^{\mathrm{m}} 54^{\mathrm{s}}, \delta=-60^{\circ} 40^{\prime} 06^{\prime \prime} ; l=314^{\circ} .71, b=\right.$ $-0.31)$, and NGC $6005\left(\alpha=15^{\mathrm{h}} 51^{\mathrm{m}} 46^{\mathrm{s}}, \delta=-57^{\circ} 17^{\prime} 30^{\prime \prime}\right.$; $\left.l=325^{\circ} .78, \quad b=-2.99\right)$. Moffat \& Vogt $(1973,1975)$ observed only six bright stars in the fields of both Pismis 18 and NGC 6005, respectively, and found no tendency to form physical groupings of stars in either case, although they suggest that observations of fainter stars are needed to better judge the physical reality of these objects. Pismis 19 was recently observed by Phelps, Janes, \& Montgomery (1994), who concluded that this cluster is significantly younger than the Hyades, based on their age parameter $\delta V$.

Based on the color-magnitude diagram (CMD) analysis, it is often possible to confirm the physical reality of objects cataloged as open cluster candidates according to their spatial appearance. Sometimes, however, independent studies lead to different results, owing to reasons such as low-quality photometric data, poor statistics of the observed fields, and the well-known problem of performing classical photoelectric photometry in crowded fields (see, e.g., Dawson 1978; Carraro \& Patat 1995).

In this paper, we present for the first time $B V I$ CCD photometry of stars in the fields of Pismis 18, Pismis 19, and NGC 6005, three open cluster candidates located nearly toward the Galactic center. In addition, we present $B V I$ CCD photometry of the old open cluster NGC 6253 $\left(\alpha=16^{\mathrm{h}} 55^{\mathrm{m}} 07^{\mathrm{s}}, \delta=-52^{\circ} 38^{\prime} 00^{\prime \prime} ; l=335^{\circ} .46, b=-6.25\right)$, whose basic parameters have recently been derived by Bragaglia et al. (1997) from a comparison of observed and synthetic CMDs and theoretical isochrones. NGC 6253 is 
located in the sky close to the above three fields and serves as a control cluster. We also present integrated CCD spectroscopy for Pismis 18, Pismis 19, and NGC 6253. These data are used to derive reddening, age, and metallicity by comparing the observed spectral features with those of wellknown Galactic and Large Magellanic Cloud clusters (Bica, Alloin, \& Santos 1990; Santos \& Bica 1993; Santos et al. 1995).

In $\S 2$, we describe the photometric and spectroscopic observations and their calibrations; in $\S 3.1$, we present the observed $(V, B-V)$ and $(V, V-I)$ diagrams and discuss their properties, while in $\S 3.2$ the CCD integrated spectra are analyzed. Section 4 summarizes the main conclusions of this study.

\section{OBSERVATIONS AND REDUCTIONS}

We have carried out CCD observations of stars in the regions of Pismis 18, Pismis 19, NGC 6005, and NGC 6253 through the $B, V$, and $I$ passbands during two observing runs in 1994 and 1995. The direct images were obtained with the 24 inch $(0.61 \mathrm{~m})$ telescope of the University of Toronto Southern Observatory, situated at Las Campanas Observatory (Chile), equipped with a Photometrics $512 \times 512$ Metachrome-coated CCD coated to yield improved blue response. As the scale on the chip is 0.45 pixel $^{-1}$, the sky area covered by a single frame is about $4^{\prime} \times 4^{\prime}$. Table 1 shows the $\log$ of observations, while schematic finding charts of the observed fields are presented in Figure 1. The air-mass values of the four observed clusters range between 1.09 and 1.28 , just lying within the air-mass range of the standard stars. The positions of the stars for which we have obtained $V$ and $V-I$ colors are marked in the charts.

Twelve standard stars from the list of Landolt (1992) were observed in each filter at the beginning, middle, and end of the night. Furthermore, a series of 10 bias exposures and 10 flat-field frames of both the twilight sky and dome were taken.

The reduction procedures followed those described by Piatti, Bica, \& Clariá (1998), using IRAF and DAOPHOT (Stetson 1991) at the Observatorio Astronómico de la Universidad Nacional de Córdoba. Atmospheric extinction was removed with the coefficients from Vázquez et al. (1996; $\left.k_{v}=0.20, k_{b}=0.31, k_{i}=0.07\right)$. The relations adopted between the instrumental and standard colors and magnitudes are the following:

$$
\begin{gathered}
v_{j, n}=v_{1}+V+v_{2}(B-V), \\
b_{k, n}=b_{1}+V+(B-V)+b_{2}(B-V), \\
i_{l, n}=i_{1}+V-(V-I)+i_{2}(V-I),
\end{gathered}
$$

where $b, v$, and $i$ represent instrumental magnitudes outside the atmosphere and $V, B-V$, and $V-I$ are the standard magnitude and colors. It should be noted that for each night $n$, the number of frames in the different filters is the same as that of the observed standard stars, so that equations (1)-(3) were fitted by least squares simultaneously. Table 2 lists the resulting coefficient values for each night. In equations (1)-(3), 0.010, 0.009, and 0.011 are the mean rms errors affecting the calibrations. By inverting these equations we could convert the instrumental color and magnitudes of the measured stars into the standard $B V I$ Johnson-Cousins system. We obtained three independent $V, B-V, V-I$ table sets, together with the $V$-frame coordinates $(X$ and $Y)$ and the instrumental DAOPHOT ALLSTAR rms errors for each field. After applying appropriate offsets to the coordinates, we carried out a cross identification among the tables corresponding to each field, averaging $V, B-V$, and $V-I$ values. This results in better sets of colors and magnitude values than those based on a single measure. Therefore, we could minimize possible anomalous values caused by the presence of contamination

\begin{tabular}{|c|c|c|c|c|}
\hline Object & $\begin{array}{l}\text { Date } \\
\text { (UT) }\end{array}$ & Filter & $\begin{array}{l}\text { Exposures } \\
\text { (s) }\end{array}$ & $\begin{array}{l}\text { FWHM } \\
\text { (arcsec) }\end{array}$ \\
\hline \multicolumn{5}{|c|}{ Photometric Data } \\
\hline \multirow[t]{3}{*}{ Pismis $18 \ldots \ldots}$. & 1995 Jun 29 & $B$ & $1 \times 400,1 \times 600$ & 1.3 \\
\hline & & $V$ & $1 \times 60,2 \times 180$ & 1.3 \\
\hline & & $I$ & $1 \times 7,2 \times 30$ & 1.2 \\
\hline \multirow[t]{3}{*}{ Pismis 19 ....... } & 1995 Jun 29 & $B$ & $1 \times 60,1 \times 900$ & 1.3 \\
\hline & & $V$ & $1 \times 60,2 \times 600$ & 1.3 \\
\hline & & $I$ & $1 \times 30,2 \times 150$ & 1.3 \\
\hline \multirow[t]{3}{*}{ NGC $6005 \ldots . .}$. & 1995 Jul 1 & $B$ & $2 \times 900$ & 1.4 \\
\hline & & $V$ & $1 \times 200,2 \times 600$ & 1.4 \\
\hline & & I & $1 \times 5,2 \times 60$ & 1.4 \\
\hline \multirow{3}{*}{ NGC $6253 \ldots \ldots$} & 1994 Sep 3 & $B$ & $1 \times 60,2 \times 600$ & 1.4 \\
\hline & & $V$ & $1 \times 60,1 \times 200,1 \times 300$ & 1.4 \\
\hline & & $I$ & $1 \times 8,1 \times 15,1 \times 30$ & 1.5 \\
\hline \multicolumn{5}{|c|}{ Spectroscopic Data } \\
\hline \multirow[t]{3}{*}{ Pismis $18 \ldots \ldots}$. & 1995 May 24 & Red & $4 \times 900$ & 2.3 \\
\hline & 1995 May 25 & Blue & $4 \times 900$ & 2.0 \\
\hline & 1995 May 30 & Red & $2 \times 900$ & 2.2 \\
\hline \multirow[t]{3}{*}{ Pismis $19 \ldots \ldots}$. & 1995 May 24 & Red & $4 \times 900$ & 2.2 \\
\hline & 1995 May 25 & Blue & $4 \times 900$ & 2.0 \\
\hline & 1995 May 30 & Red & $2 \times 900$ & 2.2 \\
\hline \multirow{3}{*}{ NGC $6253 \ldots . .}$. & 1995 May 24 & Red & $4 \times 900$ & 2.2 \\
\hline & 1995 May 25 & Blue & $4 \times 900$ & 2.0 \\
\hline & 1995 May 30 & Red & $2 \times 900$ & 2.2 \\
\hline
\end{tabular}

TABLE 1

JOURNAL OF OBSERVATIONS 


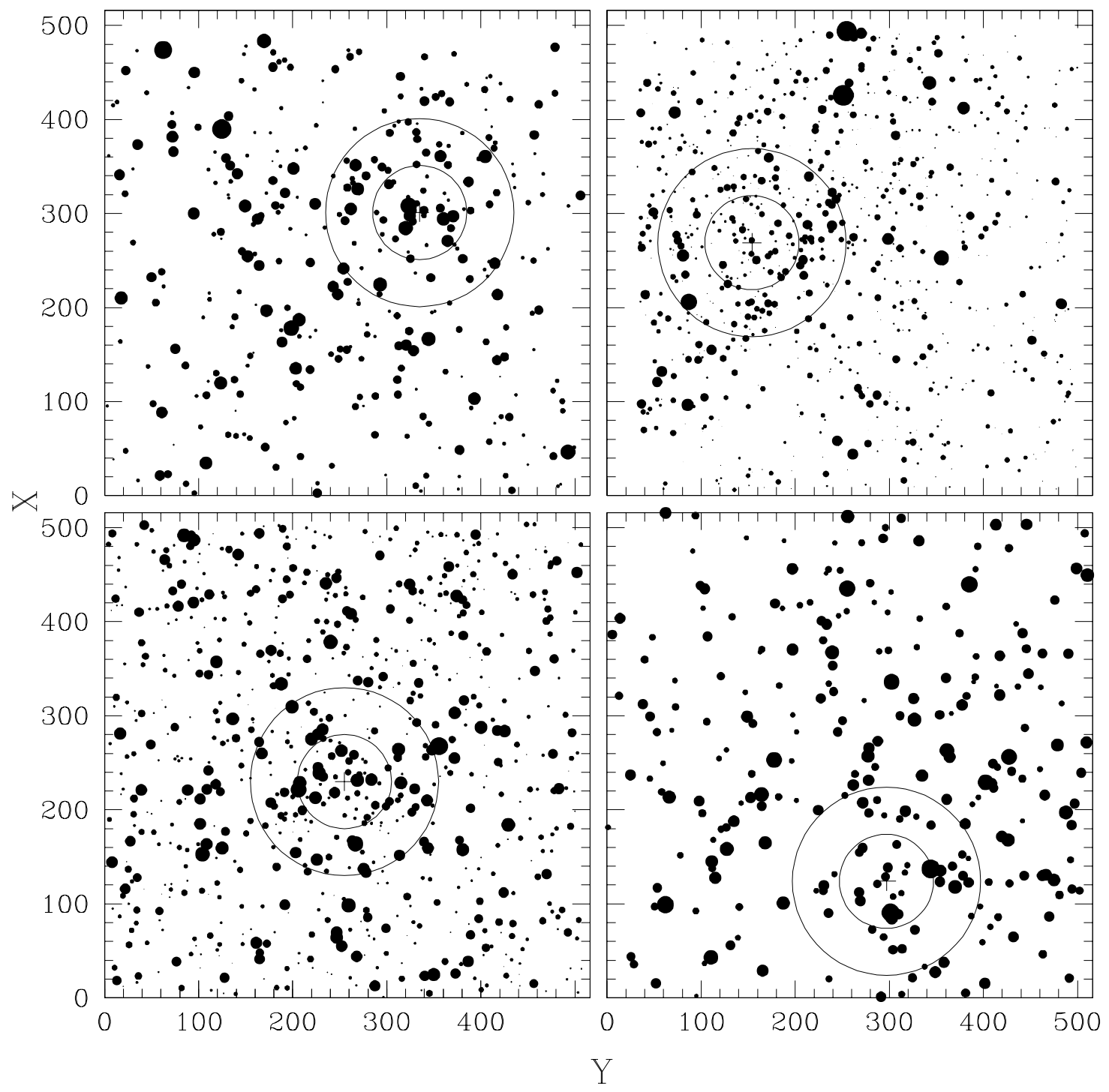

FIG. 1.-Schematic finding charts for the fields of Pismis 18 (top left), Pismis 19 (top right), NGC 6005 (bottom left), and NGC 6253 (bottom right). A cross indicates the adopted cluster center. Two concentric circles of $22^{\circ} .5$ and $45^{\circ} .0$ radii are also shown. North is up, and east is to the left.

(e.g., cosmic rays) for stars with more than one measure. Photometric internal errors were also estimated by following this procedure. Typically, $\sigma(V) \leq 0.02 \mathrm{mag}$ and $\sigma(B-V) \approx \sigma(V-I) \leq 0.03 \mathrm{mag}$ for $V<15 \mathrm{mag}$ increased to $0.04,0.06$, and 0.05 at $V \approx 18$, respectively. The resulting $V$ versus $B-V$ and $V$ versus $V-I$ diagrams are shown in Figures $2 a$ and $2 b$. A comparison of our CCD data for three well-isolated stars in Pismis 18 with photoelectric measurements by Moffat \& Vogt (1975) yields $\Delta V=-0.014 \pm 0.041$ and $\Delta(B-V)=0.002 \pm 0.013$. The agreement is then excellent, indicating that our photometry does not present systematic errors. The difference between the zero-point coefficients for the 1994 and 1995 observing runs arises from the different ZMAG parameter values used in the PHOTPARS routine, both data sets being reduced by three of the authors independently.

The integrated spectroscopic observations were carried out with the $2.15 \mathrm{~m}$ telescope at the Complejo Astronómico El Leoncito (CASLEO, Argentina) during a run in 1995 May. A CCD camera containing a Tektronix chip of $1024 \times 1024$ pixels attached to a REOSC spectrograph was utilized, the size of each pixel being $24 \mu \mathrm{m} \times 24 \mu \mathrm{m}$. All the integrated spectra were obtained by scanning the slit across the cluster. Since the total field along the slit was 4.7, we could sample background regions. A grating of 300 lines $\mathrm{mm}^{-1}$ in two different setups, namely, "blue nights" and

TABLE 2

Transformation CoEFFicients

\begin{tabular}{ccccccc}
\hline \hline Date (UT) & $b_{1}$ & $b_{2}$ & $v_{1}$ & $v_{2}$ & $i_{1}$ & $i_{2}$ \\
\hline 1994 Sep 3 ...... & $6.357 \pm 0.012$ & $-0.016 \pm 0.006$ & $6.027 \pm 0.007$ & $-0.040 \pm 0.005$ & $5.283 \pm 0.013$ & $-0.039 \pm 0.007$ \\
1995 Jun 29..... & $7.267 \pm 0.009$ & $-0.043 \pm 0.004$ & $7.051 \pm 0.009$ & $-0.039 \pm 0.010$ & $6.551 \pm 0.011$ & $-0.084 \pm 0.011$ \\
1995 Jul 1 ....... & $7.244 \pm 0.010$ & $-0.073 \pm 0.005$ & $7.014 \pm 0.009$ & $-0.022 \pm 0.009$ & $6.532 \pm 0.012$ & $-0.090 \pm 0.012$ \\
\hline
\end{tabular}



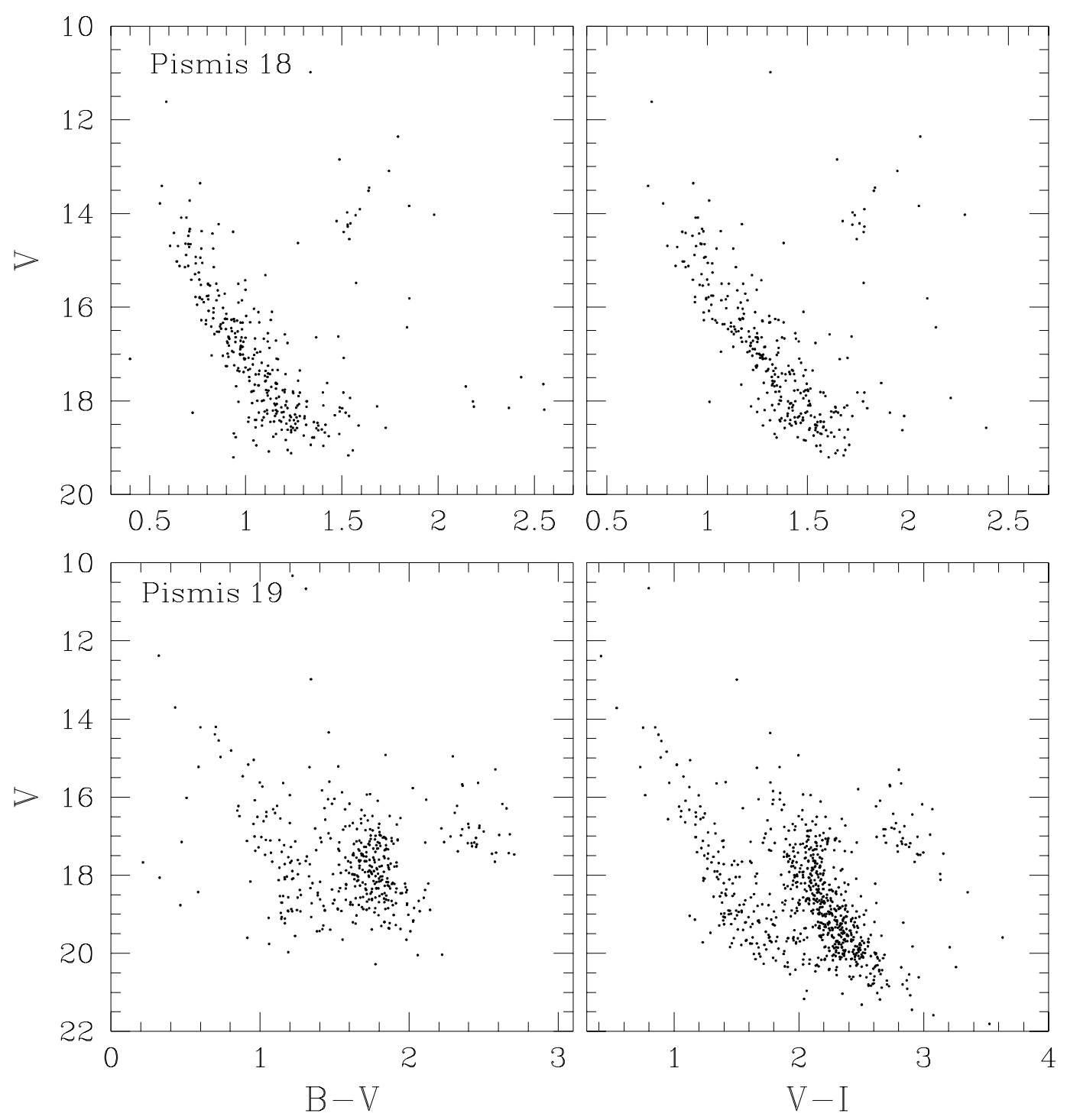

Fig. 2. $-B-V$ and $V-I$ vs. $V$ CMDs of stars in the fields of Pismis 18, Pismis 19, NGC 6005, and NGC 6253

"red nights," was used. During the blue nights, spectra ranging from 3500 to $7000 \AA$, with an average dispersion in the observed region of $140 \AA \mathrm{mm}^{-1}$, or $3.46 \AA$ pixel $^{-1}$, were obtained. According to the FWHM of the HeAr comparison lamps, the slit width was 4.2 , thus resulting in an average resolution of $14 \AA$. On the red nights, the range was 5800-9200 $\AA$ with a similar dispersion $\left(3.36 \AA\right.$ pixel $\left.^{-1}\right)$. The slit width was 4."2, resulting in an average resolution of 17 $\AA$. In order to eliminate the second-order contamination, an OG 550 filter was employed.

Series of 15 minute exposures were taken for Pismis 18, Pismis 19, and NGC 6253, yielding a total of 60 and 90 minutes for the blue and the red spectra, respectively. Unfortunately, it was not possible to obtain any integrated spectrum of NGC 6005. In addition to the observations of the program fields, spectrophotometric standards were measured to derive flux calibrations. Stars LTT 4364, EG 274, and LTT 7379 (Stone \& Baldwin 1983) were used in the blue range. In the red range, the hot dwarf star HD 160233 (Gutiérrez-Moreno et al. 1988) was added in order to correct for telluric absorption bands. We also obtained bias, dome, and twilight sky and tungsten lamp flat fields, as well as frames of HeAr comparison lamps between or after object observations.

The data were reduced in the standard way using IRAF and the computing facilities at the Observatorio Astronómico de la Universidad Nacional de Córdoba. Briefly, in order to correct the frames for high and low spatial frequency variations, we subtracted the bias and used flat-field frames previously combined. Then, background sky subtractions were performed using pixel rows from the same frame after cleaning cosmic rays from the background sky regions. We checked for the absence of significant background sky residuals on the resulting spectra. The cluster spectra were extracted and wavelength-calibrated by fitting observed HeAr comparison lamp spectra with template spectra. The rms errors involved in these calibrations are $0.72 \AA(0.21$ pixels $)$ for the blue nights and $0.39 \AA(0.12$ pixels) for the red ones. Extinction corrections and flux calibrations derived from the observed standard stars were applied to the cluster spectra. Last, the telluric absorption bands in the near-IR were eliminated by means of the procedures outlined in Bica \& Alloin (1987). Figure 3 presents the calibrated integrated spectra of Pismis 18, Pismis 19, 

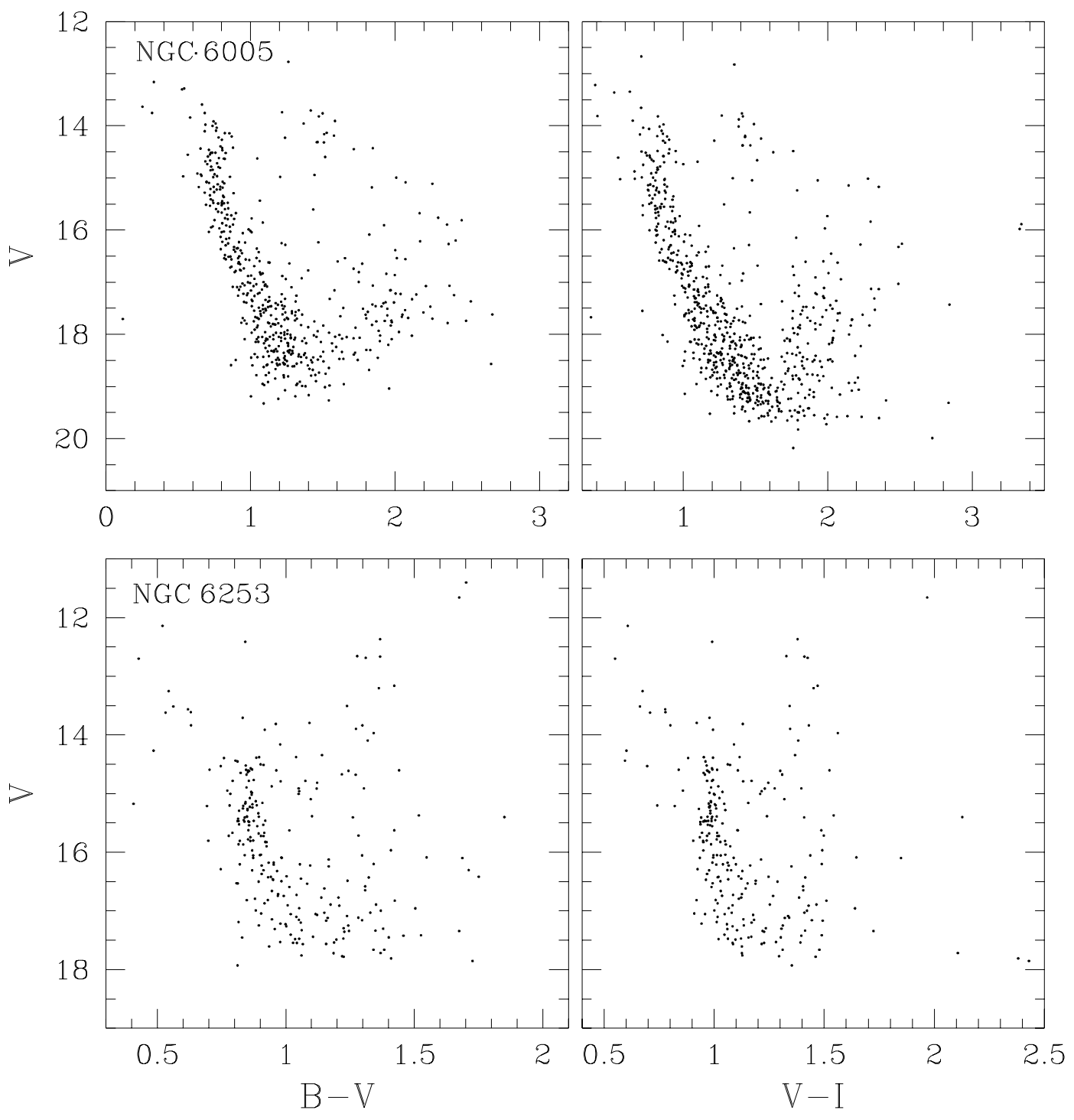

Fig. 2.-Continued

and NGC 6253 normalized to $F_{\lambda}=1$ at $\lambda=6000 \AA$. Indeed, a certain amount of field star contamination, mainly from stars located close to the cluster center, may be expected. However, only bright field stars could affect the spectra significantly, and this was not the case in any of the three observed clusters. This means that the astrophysical information of the resulting spectra is essentially the same as that obtained from the circular extractions (see § 3.1).

\section{ANALYSIS AND DISCUSSION}

\subsection{Photometric Data}

An inspection of the observed CMDs (Figs. $2 a$ and $2 b$ ) allows us to distinguish several morphological features:

1. The observed $(V, V-I)$ diagrams reveal, in general, more stars than those including the $B$ passband. This is particularly evident for the fields of Pismis 19 and NGC 6005 , wherein an increase of faint stars is clearly seen. This increase is not only due to reddening effects but also to the crowdedness of the fields.

2. The two CMDs of Pismis 18 reveal well-defined star sequences with relatively small field star contamination, while the CMDs of the remaining three fields are clearly more affected by the presence of field stars. In particular, a parallel sequence of field stars is clearly seen in Pismis 19. Note that the main features of the CMDs of Pismis 19 are also clearly visible in the $(V, V-I)$ diagram of Phelps et al. (1994).

3. The global morphology of the observed CMDs of Pismis 18, Pismis 19, and NGC 6005 resemble those of intermediate-age open clusters in which the turnoff mass and the mass of the red giant stars are large enough to allow a mild $\mathrm{He}$ core ignition, thereby avoiding the formation of a developed giant branch (Barbaro \& Pigatto 1984). Instead, red giant clumps (RGCs) are clearly visible centered at about $V \approx 14.3, V-I \approx 1.75 ; V \approx 17.2, V-I \approx 2.8$; and $V \approx 14.2, V-I \approx 1.4$ in Pismis 18 , Pismis 19 , and NGC 6005 , respectively. Possible blue stragglers could be present in the CMDs of Pismis 19 and NGC 6005.

4. The overall morphology of the CMDs of NGC 6253 bears a close resemblance to those of old open clusters. Its two CMDs show a clear main sequence (MS), slightly contaminated by field stars at the faintest magnitudes, a subgiant branch, a poorly defined red giant branch extending 


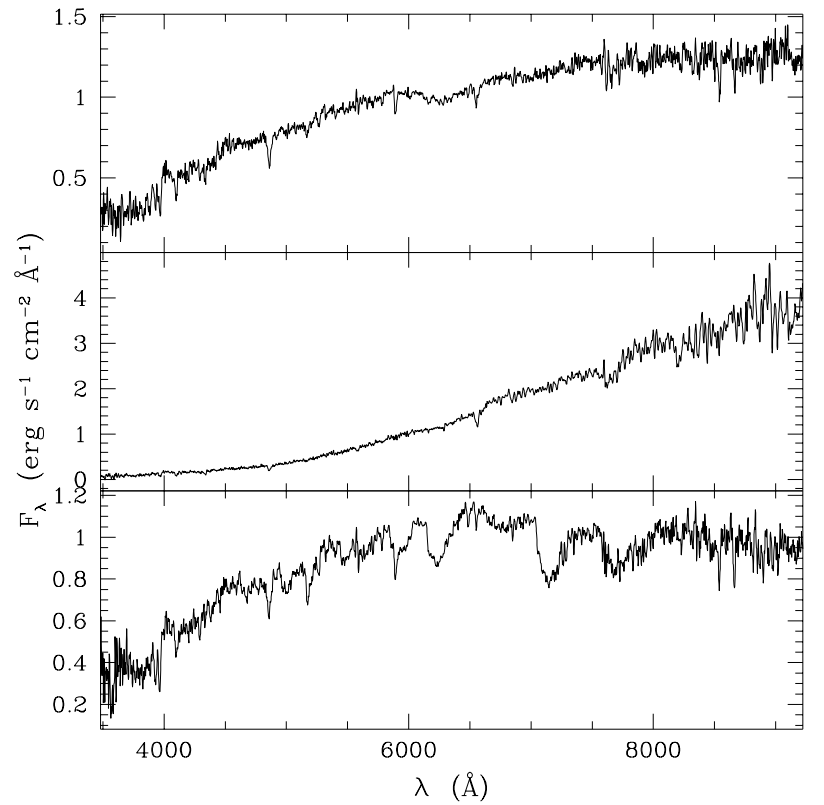

Fig. 3.-Observed integrated spectra in absolute flux units: Pismis 18 (top), Pismis 19 (middle), and NGC 6253 (bottom).

from $V \sim 12.5$ to about $V \sim 15 \mathrm{mag}$, and a sparsely populated red giant branch. We also note the presence of a small number of stars populating the top left part of the CMDs, which are likely to be blue stragglers. A comparison of the present CMDs of NGC 6253 with those obtained by Bragaglia et al. (1997) using the Danish $1.54 \mathrm{~m}$ telescope (La Silla, Chile) indicates that no significant additional information is provided by MS stars fainter than $V \sim 18 \mathrm{mag}$, which is precisely the present limiting magnitude for this cluster.

In order to obtain a better definition of the CMDs for the four observed clusters by eliminating possible nonmember stars in each field, we first determined the corresponding cluster centers. This was accomplished by building the stellar density profiles as a function of pixel bins in the $x$ and $y$-directions, using all the stars in Figures $2 a$ and $2 b$. The adopted final coordinates for the cluster centers are $\left(X_{c}, Y_{c}\right)=(301,335),(269,154),(230,255)$, and $(124,297)$ for Pismis 18, Pismis 19, NGC 6005, and NGC 6253, respectively. We then extracted $(V, B-V)$ and $(V, V-I)$ diagrams around $\left(X_{c}, Y_{c}\right)$ for $r<22.5$ (50 pixels) and $r<45^{\prime \prime} .0$ (100 pixels). The smallest circular extractions allow us to know which are the CMD regions where the fiducial cluster sequences are located, while the largest circular extractions represent a compromise between minimizing the unavoidable field star contamination and maximizing the number of cluster stars. The resulting CMDs for the four selected fields are shown in Figures $4 a$ and $4 b$. Stars located within 50 and 100 pixel-wide annuli from the cluster centers are shown as large circles and crosses, respectively, whereas dots represent the remaining stars.

As shown in Figure $4 a$ (top), no additional information is provided by the CMDs of Pismis 18, wherein the cluster MS is well defined for at least $4 \mathrm{mag}$ fainter than the turnoff. The circular extractions also reveal the presence of several red cluster giants. On the other hand, the circular extractions allow us to clearly identify the MS for Pismis 19. In fact, Figure $4 a$ (bottom) reveals that the most crowded sequence is mainly populated by cluster stars, while the blue sequence represents the distribution of foreground stars along the line of sight affected by different amounts of interstellar absorption (see below). Phelps et al. (1994) argued that the sequence of hot, blue stars likely represents stars from the nearby young open cluster NGC 5617, located approximately $35^{\prime}$ from Pismis 19 . We note, however, that the open cluster Trumpler 22 is even closer in the sky to Pismis 19 than is NGC 5617. Note also that the blue sequence is also visible for stars located within 2.0 of the center of Pismis 19 (see Fig. 27 of Phelps et al. 1994). These stars appear to follow the direction of the reddening vector rather than the typical curvature of a young open cluster MS. The argument that the stars observed in some limited region of the sky show a general appearance in the CMD rather similar to a MS is frequently explicitly or implicitly presented as a proof of the physical reality of a cluster. However, even field stars show the appearance of an open star cluster in the CMDs. Burki \& Maeder (1973) showed that field dwarf sequences have a lower envelope, which does not depend on the space density of stars, but on the parameters of interstellar extinction, namely, $R \equiv A_{v} / E(B-V)$. They also found that this sequence has a smaller curvature than the zero-age main sequence (ZAMS).

The extracted CMDs of NGC 6005 (Fig. 4b, top) show a populated cluster MS with a well-defined turnoff hook. According to the position of the RGC, NGC 6005 appears to have an age nearly similar to those of Pismis 18 and Pismis 19. A few stars located in the top left part of the CMDs are likely blue stragglers.

$\mathrm{Ng}$ et al. (1996) have recently studied the contribution of stars from the disk and bulge population to CMDs of star fields located at low Galactic latitudes in the direction of the Galactic center. They have also identified the regions of the CMDs where the disk and bulge stars are located. According to these findings, the sequence of red stars at about $V-I \approx 2.0$ in the $(V, V-I)$ diagram of NGC 6005 should correspond to evolved stars of an intermediate-age/old disk population component. $\mathrm{Ng}$ et al.'s (1996) results also show that the slightly tilted blue sequence in the CMDs of Pismis 19 is formed by MS disk stars.

Figure $4 b$ (bottom) reveals that the stars in the core region of NGC 6253 are mainly upper-MS stars. The subgiant and red giant stars appear to be spread out in the outer cluster regions. The fact that evolved stars in old open clusters are, in general, less centrally concentrated than are the brightest stars remaining on the upper MS is supported not only from observational evidence (see, e.g., Hawarden 1975; Mathieu 1984), but also from models of dynamical evolution of open clusters (see, e.g., Wielen 1967; Terlevich 1987). It has long been suggested that this phenomenon is a consequence of mass loss during the most luminous phases, as well as mass segregation during the dynamical cluster evolution.

To estimate mean cluster reddenings and apparent distance moduli, we matched the extracted $(V, B-V)$ and $(V$, $V-I$ ) diagrams to the ZAMS of Schmidt-Kaler (1982) and Piatti, Clariá, \& Bica (1998), respectively. Table 3 lists the resulting $E(B-V)$ and $E(V-I)$ color excesses and apparent distance moduli, together with the corresponding errors estimated on the basis of the MS width. These values have been adopted for the subsequent analysis. From both color excesses, a mean value for the ratio $E(V-I) /$ $E(B-V)=1.26 \pm 0.10$ was derived, thus indicating that the 

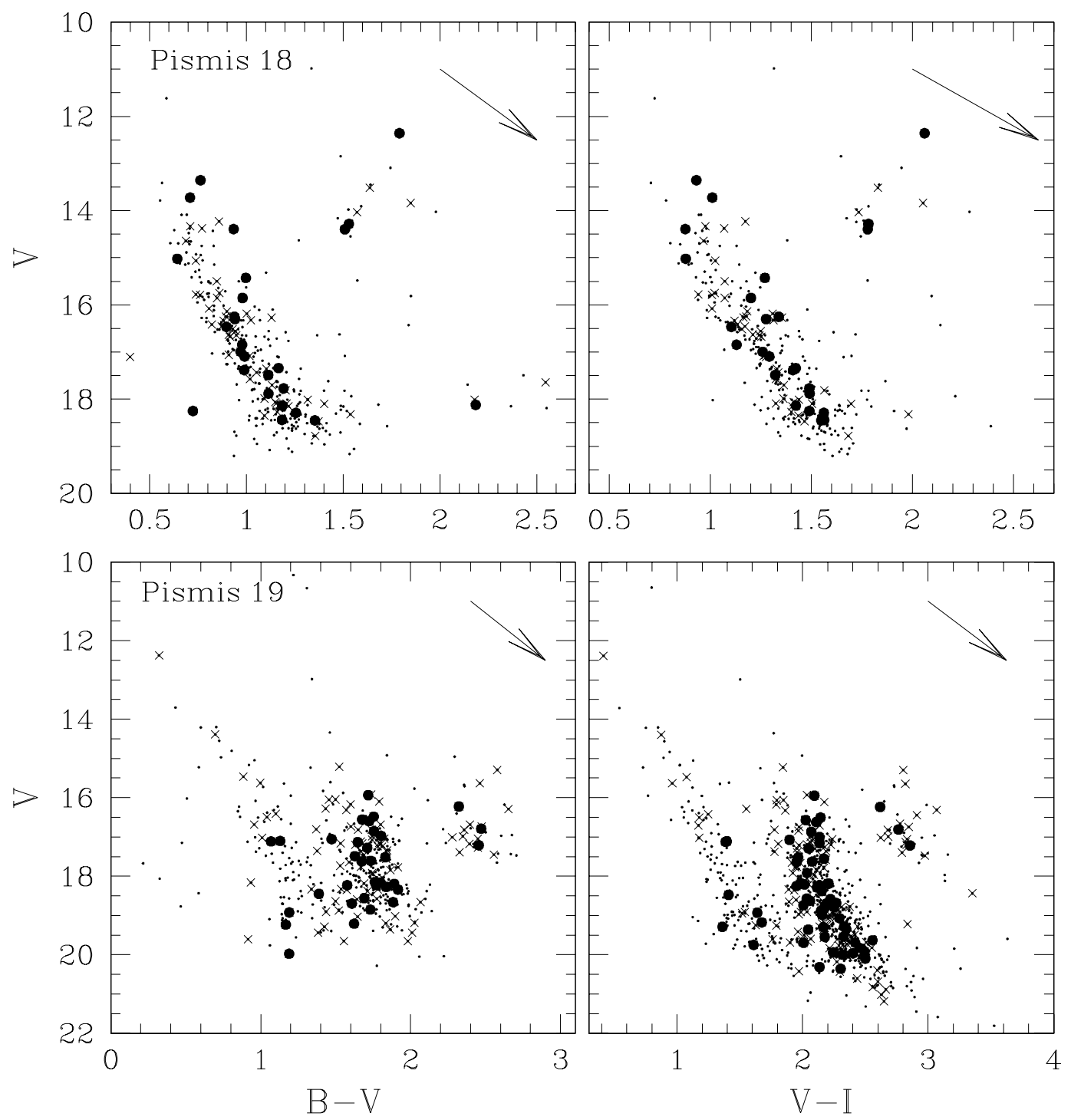

Fig. 4. - CMDs of stars in the fields of Pismis 18, Pismis 19, NGC 6005, and NGC 6253. All measured stars (dots), circular extraction for $r<22$ ".5 (large circles), and $r<45.0$ (crosses) are superposed. Reddening vectors with $\Delta E(B-V)=0.5$ are also shown.

interstellar absorption in the direction of the studied clusters follows approximately the normal extinction law. In the subsequent analysis, we have therefore adopted the values 1.25 and 3.0 for the $E(V-I) / E(B-V)$ and $R=A_{v} / E(B-V)$ ratios, respectively (Turner 1976; Walker 1985; Straižys 1992).

Piatti et al. (1998) have recently calibrated the $M_{v}$ versus $(V-I)_{0}$ diagram as a function of age, using high-quality CCD VI data of template open clusters with a wide age range. Such a diagram is very useful to derive ages of highly reddened open clusters regardless of their metal content, since the $(V-I)_{0}$ index is virtually free of metallicity effects (see, e.g., Rosvick 1995). Kassis, Friel, \& Phelps (1996), for example, succesfully fitted theoretical isochrones of $5 \mathrm{Gyr}$ with $[\mathrm{Fe} / \mathrm{H}]=-0.23,-0.45$ and -0.75 to the observed $(V, V-I)$ diagram of the open cluster ESO 092-SC18.

To estimate ages of the present cluster sample, we have applied reddening and distance corrections to the observed CMDs using the adopted $E(V-I)$ and distance values. We then fitted the empirical isochrones of Piatti et al. (1998) to

TABLE 3

Cluster Fundamental Parameters Derived from Analysis of the CMDs

\begin{tabular}{ccccr}
\hline \hline Cluster & $\begin{array}{c}E \\
(B-V)\end{array}$ & $\begin{array}{c}E \\
(V-I)\end{array}$ & $V-M_{v}$ & \multicolumn{1}{c}{$\begin{array}{c}\text { Age } \\
(\mathrm{Gyr})\end{array}$} \\
\hline Pismis 18 ...... & $0.50 \pm 0.05$ & $0.70 \pm 0.05$ & $13.25 \pm 0.25$ & $0.9 \pm 0.3$ \\
Pismis 19 ...... & $1.45 \pm 0.10$ & $1.85 \pm 0.10$ & $16.25 \pm 0.50$ & $1.1 \pm 0.3$ \\
NGC 6005..... & $0.45 \pm 0.05$ & $0.50 \pm 0.05$ & $13.50 \pm 0.25$ & $1.2 \pm 0.3$ \\
NGC 6253..... & $0.20 \pm 0.05$ & $0.25 \pm 0.05$ & $11.50 \pm 0.25$ & $\sim 5.0 \pm 1.0$ \\
\hline
\end{tabular}



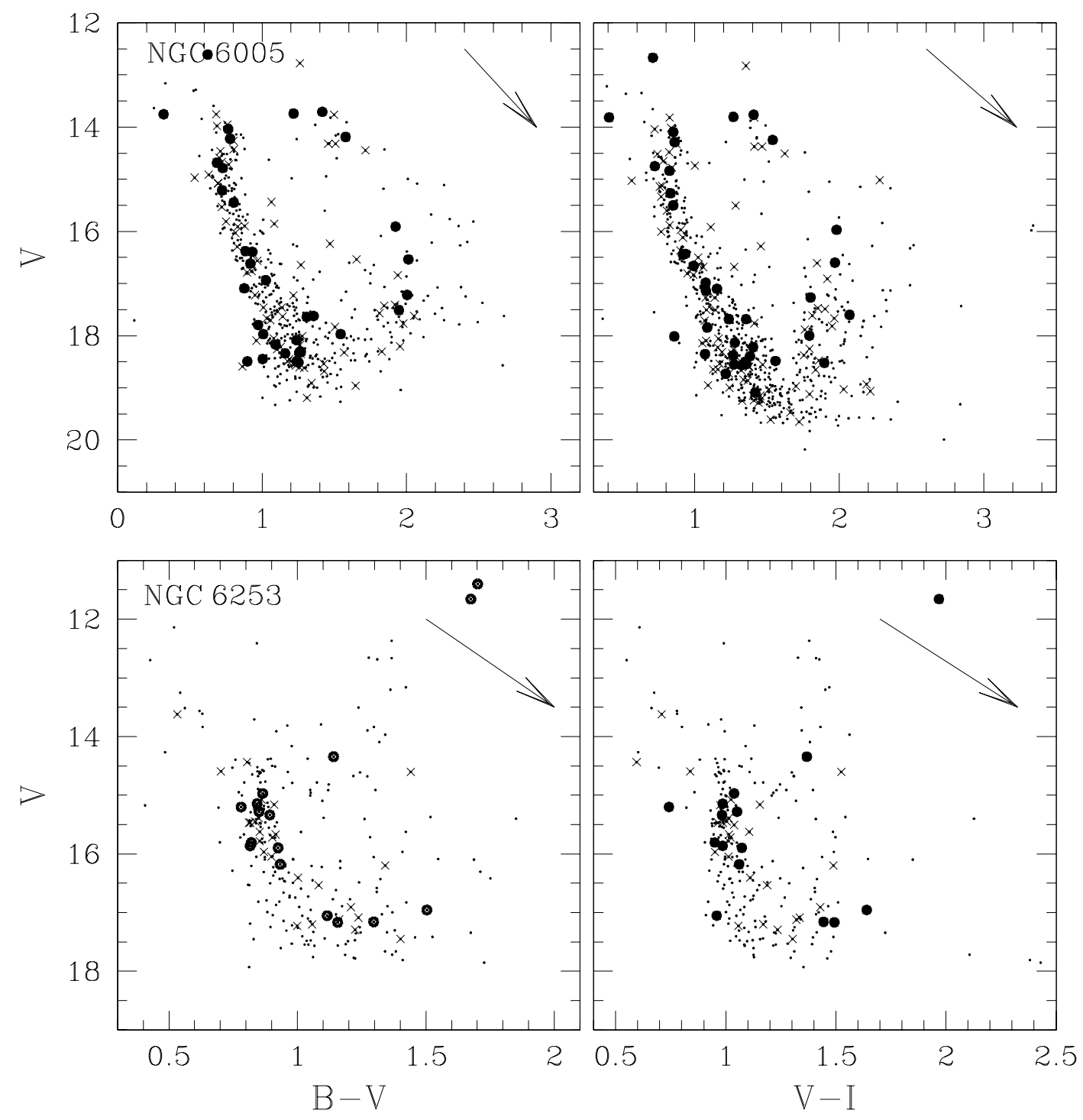

FIG. 4.-Continued

the cluster $M_{v}$ versus $(V-I)_{0}$ diagrams and interpolated between the isochrones to derive ages. These estimations were performed taking into account the positions of the MS and RGC stars distributed within 100 pixel-wide annuli from the cluster centers. The rightmost column in Table 3 lists the resulting cluster ages and their uncertainties estimated from the $E(V-I)$ and $V-M_{v}$ errors, whereas Figure 5 shows the $M_{v}$ versus $(V-I)_{0}$ diagrams with the isochrones superposed. The boxed regions in Figure 5 represent the loci of the RGCs of the template open clusters NGC 3680 and IC $4651(t \approx 2.0 \mathrm{Gyr})$. In Figure 5, we have included all the measured stars of each field.

\subsection{Spectroscopic Data}

Integrated spectra of star clusters allow one to determine their basic parameters, such as reddening, age, and metallicity. Bica \& Alloin (1986a, 1987, hereafter BA86a and BA87, respectively) have studied integrated spectra in the visible and near-IR ranges of Galactic open and globular clusters, as well as Magellanic Cloud clusters. They examined the behavior of metallic and Balmer line equivalent widths, as well as the continuum energy distribution in the spectral range $3700-10000 \AA$. They also generated a library of template cluster spectra with well-known properties.

We determined the fundamental parameters of the clusters from their integrated spectra using the SPEED spectral analysis software (Schmidt 1988) at the Observatorio Astronómico de la Universidad Nacional de Córdoba. A direct age estimate was first obtained from equivalent widths of the Balmer lines in absorption in each spectrum by interpolating these values in the age calibration of Bica \& Alloin (1986b, hereafter BA86b). The ages derived by this method are reddening independent. We then selected an appropriate set of template spectra according to the ages provided by the Balmer lines and derived cluster reddening by matching each observed spectrum to that of the template that most resembles it, thus making use of the full spectral distribution.

In order to measure the equivalent widths of the Balmer lines, we have taken into account both the spectral windows and flux points as defined by BA86a and BA86b. The flux points were fitted to the corresponding local continuum with spline functions. The errors affecting the derived equivalent widths were estimated from different measurements of 


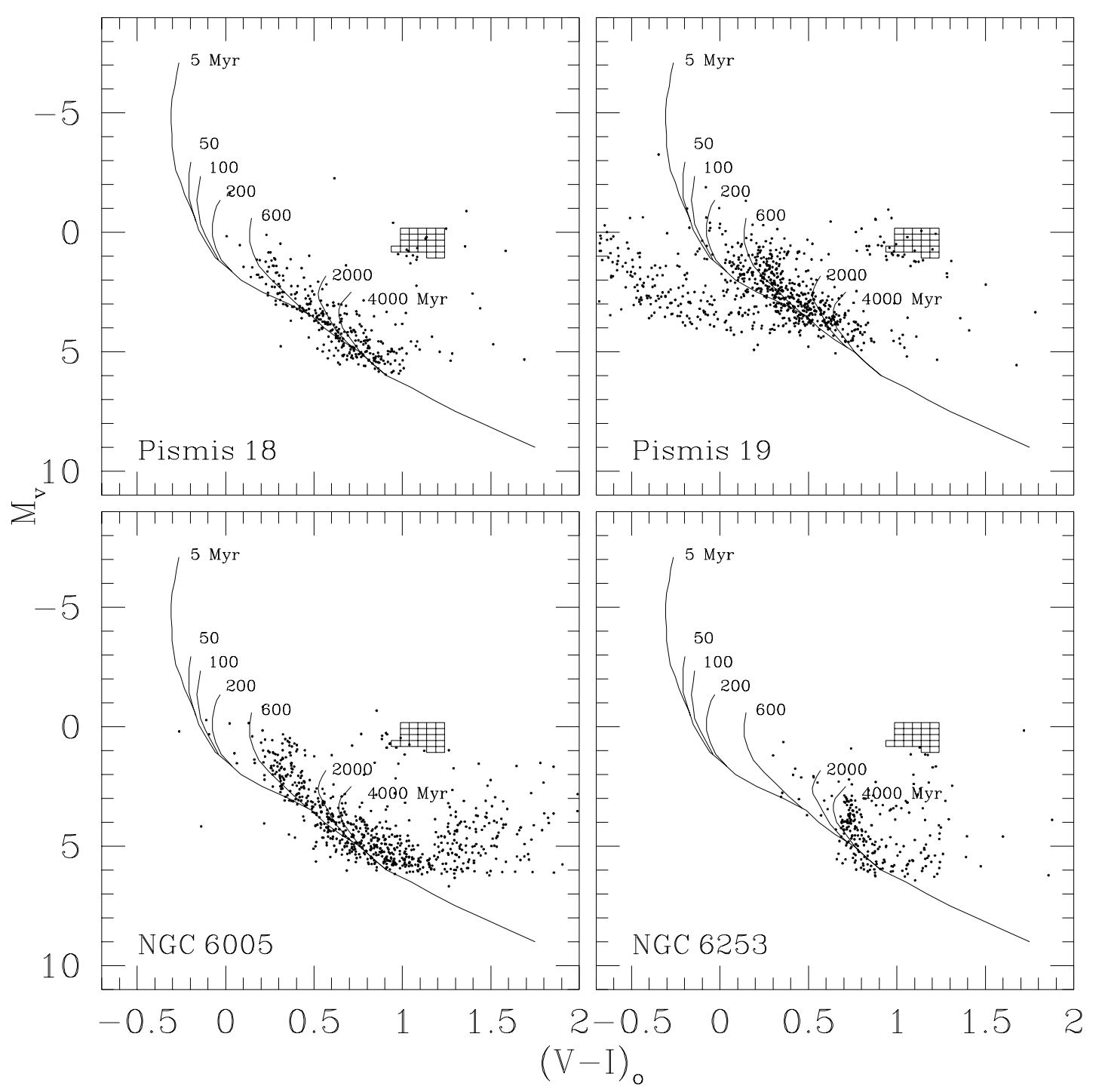

FIG. 5. $-M_{v}$ vs. $(V-I)_{0}$ diagrams with the isochrones of Piatti, Clariá, \& Bica (1998) superposed

the Balmer lines using high and low continuum tracings in order to take into account the spectral noise. The resulting uncertainties in the equivalent widths are smaller than those associated with the intrinsic dispersions in Table 2 of BA86b. Column (6) in Table 4 lists the mean age and its error obtained from the average of ages provided by the $\mathrm{H} \alpha$ to $\mathrm{H} \delta$ Balmer lines.

The template cluster spectra were taken from Bica (1988). They are average spectra of the Large Magellanic Cloud and Galactic star clusters grouped according to their evolutionary stages. Unfortunately, only a few template spectra are available for the intermediate age range. For the selection of the template spectra, we used the reddeningindependent ages obtained previously as a first guess. The template groups that were useful in the present analysis are I1 (age $t \approx 1.0 \mathrm{Gyr})$ and $\mathrm{I} 2(t \approx 2.0 \mathrm{Gyr})$. Then reddening and template were varied to obtain the best match to both the continuum and lines of the cluster spectra. The reddening corrections were performed using the interstellar absorption law derived by Seaton (1979). Columns (3) and (4) in Table 4 list the age of the template that most resembles the cluster spectrum and the derived reddening. In the

TABLE 4

Cluster Fundamental Parameters Derived from Spectroscopic Analysis

\begin{tabular}{|c|c|c|c|c|c|c|c|}
\hline \multirow[b]{2}{*}{$\begin{array}{l}\text { CLUSTER } \\
\text { (1) }\end{array}$} & \multicolumn{4}{|c|}{ EQUIVALENT WIDTH (Å) } & \multicolumn{2}{|c|}{ AGE (Gyr) } & \multirow[b]{2}{*}{$\begin{array}{c}E(B-V) \\
(8)\end{array}$} \\
\hline & $\begin{array}{l}\mathrm{H} \alpha \\
(2)\end{array}$ & $\begin{array}{c}\mathrm{H} \beta \\
(3)\end{array}$ & $\begin{array}{l}\mathrm{H} \gamma \\
(4)\end{array}$ & $\begin{array}{r}\mathrm{H} \delta \\
(5)\end{array}$ & $\begin{array}{l}\text { Balmer } \\
(6)\end{array}$ & $\begin{array}{c}\text { Template } \\
\text { (7) }\end{array}$ & \\
\hline Pismis 18 . & $4.49 \pm 0.18$ & $6.83 \pm 0.06$ & $7.06 \pm 0.42$ & $8.51 \pm 0.51$ & $1.80 \pm 0.60$ & 1.0 & 0.40 \\
\hline Pismis $19 \ldots \ldots$. & $5.07 \pm 0.35$ & $9.07 \pm 0.18$ & $6.08 \pm 0.42$ & $13.00 \pm 0.52$ & $0.75 \pm 0.25$ & 1.0 & 1.40 \\
\hline NGC $6253 \ldots . .$. & $2.74 \pm 0.08$ & $6.00 \pm 0.06$ & $1.51 \pm 0.39$ & $6.79 \pm 0.34$ & $3.00 \pm 1.40$ & $\sim 5.0$ & 0.20 \\
\hline
\end{tabular}




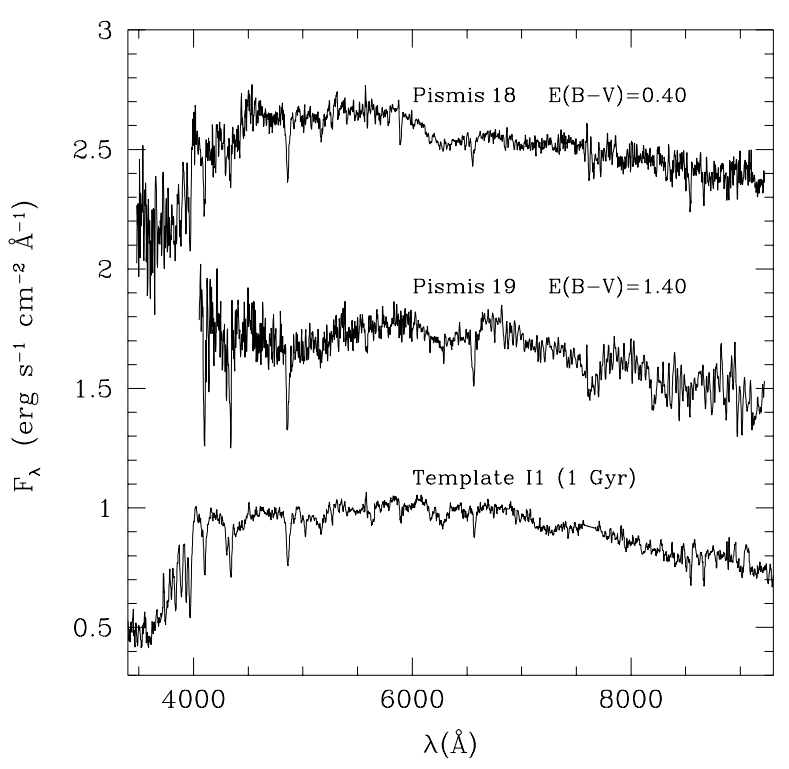

FIG. 6.- Reddening-corrected integrated spectra of Pismis 18 and Pismis 19 compared with template I1.

case of NGC 6253, a weighted age between I2 and G1 $(t \approx 10.0 \mathrm{Gyr})$ templates was adopted. The associated error in the $E(V-I)$ color excess is typically $\sim 0.05 \mathrm{mag}$.

Figure 6 illustrates the observed integrated spectra of Pismis 18 and Pismis 19 corrected for the derived foreground reddening values. These spectra were normalized to $F_{\lambda}=1$ at $\lambda=6000 \AA$ and shifted by a constant in Figure 6 for presentation purposes. Note that template I1 successfully reproduces the global and particular features observed in the spectra of Pismis 18 and Pismis 19. Although the continuum slope and intensities of the Balmer lines in general exhibit a nearly similar appearance in all spectra, small spectral differences can still be observed. It must be noted that the template spectra are the average of the integrated spectrum of several different clusters, so that they represent the stellar population synthesis of clusters within a limited age range. On the other hand, the program cluster spectra reflect the behavior of the combined light coming mainly from their members. TiO bands at $\lambda \approx 6650$ $\AA$, for example, appear to be more prominent in the spectra of Pismis 18 and Pismis 19 than in the I1 template. These features might be indicating the existence of differences between the red giant populations of the clusters and the I1 template.

Figure 7 shows the reddening-corrected spectrum of NGC 6253 compared with the I2 and G1 templates. They are normalized to $F_{\lambda}=1$ at $\lambda=6000 \AA$ and shifted by different constants. Note that the continuum distribution of

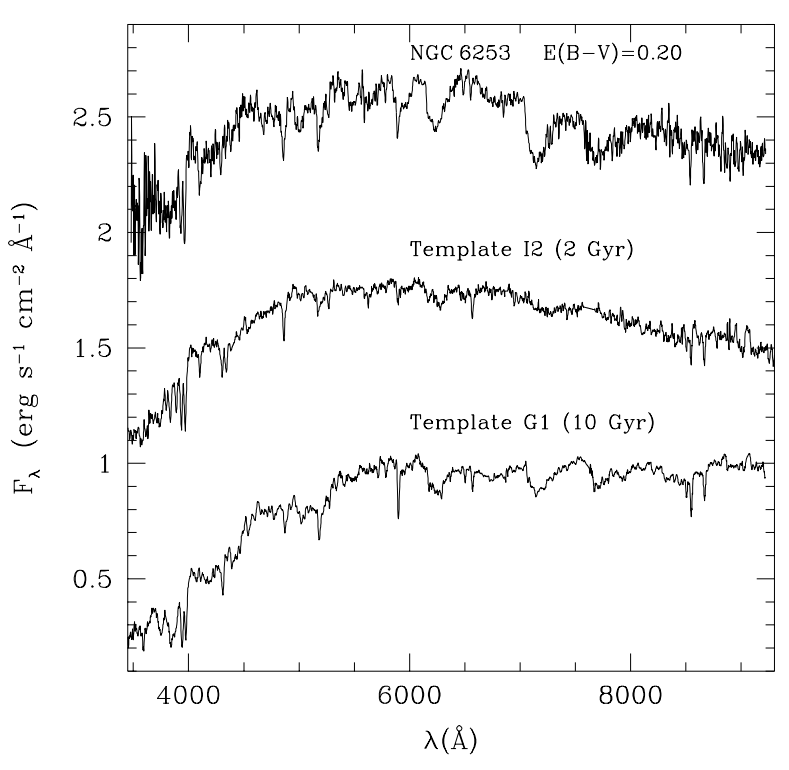

FIG. 7.- Reddening-corrected integrated spectrum of NGC 6253 compared with templates $\mathrm{I} 2$ and $\mathrm{G} 1$.

NGC 6253 toward the near-IR range is very similar to that of I2. Balmer lines resemble more those in the I2 template, which indicates the intermediate-age nature of NGC 6253. On the other hand, the spectrum of NGC 6253 presents pronounced $\mathrm{TiO}$ bands from the visible to the near-IR, which implies a stronger contribution of red giants with respect to I 2 and possibly also reflects the higher metallicity of NGC 6253. These strong TiO bands agree well with the presence of at least one M5 III MK-type star (Serote Roos, Boisson, \& Joly 1996) and an extended red giant branch (see Fig. $4 b$ ).

We measured the equivalent widths $(W)$ of the $\mathrm{Ca}$ II triplet lines using the regions around $\lambda=8408 \AA$ and $\lambda=8700 \AA$ (BA87) as continuum points. Table 5 presents the window limits and the $W$ 's. The error assigned to each measurement was calculated by considering local high and low continuum tracings. Although the correlations between the $W$ of the Ca II triplet lines with metallicity are basically single valued for integrated spectra of star clusters (BA87), there are sources of scatter that affect the determination of $Z$. These uncertainties are related to the different stellar components contributing to the integrated light rather than to the errors arising from the quality of the spectrum itself. The contamination by TiO bands and Paschen lines affects the $\mathrm{Ca}$ II windows. TiO may reinforce the metallicity dependence of the Ca II triplet (especially for the 8498 and $8542 \AA$ lines), while Paschen lines introduce a systematic increase for blue clusters whatever their metallicities are. The

TABLE 5

EQUIVALENT WIDTHS FOR THE Ca II WINDOWS

\begin{tabular}{ccccc}
\hline \hline \multirow{2}{*}{$\begin{array}{c}\text { Window } \\
(\AA)\end{array}$} & & \multicolumn{3}{c}{ EQuivalent WIDTH $(\AA \lambda)$} \\
\cline { 3 - 5 } & ID & Pismis 18 & Pismis 19 & NGC 6253 \\
\hline $8498 / 44 \ldots \ldots .$. & Ca II + Pa16 & $0.93 \pm 0.17$ & $1.27 \pm 0.22$ & $3.10 \pm 0.12$ \\
$8542 / 44 \ldots \ldots$. & Ca II + Pa15 & $3.96 \pm 0.15$ & $2.87 \pm 0.20$ & $4.74 \pm 0.11$ \\
$8670 / 60 \ldots \ldots .$. & Ca II + Pa13 & $2.43 \pm 0.19$ & $0.26 \pm 0.24$ & $4.86 \pm 0.16$ \\
\hline
\end{tabular}


TABLE 6

Adopted Cluster Fundamental Parameters

\begin{tabular}{|c|c|c|c|c|c|}
\hline Cluster & $\begin{array}{c}E \\
(B-V)\end{array}$ & $V_{0}-M_{v}$ & $\begin{array}{c}d \\
(\mathrm{kpc})\end{array}$ & $\begin{array}{l}\text { Age } \\
\text { (Gyr) }\end{array}$ & {$[\mathrm{Fe} / \mathrm{H}]$} \\
\hline Pismis 18 & $0.50 \pm 0.05$ & $11.75 \pm 0.40$ & $2.24 \pm 0.41$ & $1.2 \pm 0.4$ & 0.0 \\
\hline Pismis $19 \ldots \ldots$. & $1.45 \pm 0.10$ & $11.90 \pm 0.80$ & $2.40 \pm 0.88$ & $1.0 \pm 0.2$ & -0.1 \\
\hline NGC $6005 \ldots \ldots$. & $0.45 \pm 0.05$ & $12.15 \pm 0.40$ & $2.69 \pm 0.49$ & $1.2 \pm 0.3$ & \\
\hline NGC $6253 \ldots . .$. & $0.20 \pm 0.05$ & $10.90 \pm 0.40$ & $1.51 \pm 0.28$ & $5.0 \pm 1.5$ & 0.2 \\
\hline
\end{tabular}

Paschen absorptions have more of an effect on the Ca II 8662 À line.

\subsection{Adopted Cluster Parameters}

To estimate the metallicity of Pismis 18 , Pismis 19 , and NGC 6253, we compared the $W(\mathrm{Ca}$ II) values with those measured by Santos \& Bica (1993) and BA87 for open clusters with independent metallicity determinations from individual stars. The sums of the equivalent widths for the three Ca II lines in Pismis 18, Pismis 19, and NGC 6253 are $\sum W=7.32 \pm 0.51,4.40 \pm 0.66$, and $12.70 \pm 0.39$, respectively. These values compare well with the $W$ 's of $9.6,4.9$, and 13.2 for NGC 6067, 6231, and 6705, whose [Fe/H] values are 0.01 (Luck 1994), -0.1 (Kilian, Montenbruck, \& Nissen 1994), and 0.21 (Thogersen, Friel, \& Fallon 1993), respectively. We thus conclude that Pismis 18 has nearly solar metal content, while Pismis 19 and NGC 6253 appear to be slightly metal-poor and metal-rich, respectively (see Table 6).

The adopted basic parameters for the present cluster sample were obtained by combining those derived from CMDs and the spectroscopic analysis. The $E(B-V)$ color excesses were averaged, assigning double weight to the photometric values. This criterion was adopted because the photometric $E(B-V)$ color excess is a more reliable indicator of the mean total absorption affecting the cluster. Nevertheless, both reddening determinations show very good agreement. Of course, in the case of NGC 6005 we have adopted the results provided by the photometric analysis. The cluster ages correspond basically to the average of three independent estimates, namely, the age obtained from the isochrone fitting, the age derived from the Balmer line equivalent widths, and that of the template spectrum used to derive cluster reddening. These also agree well. Finally, cluster distances $(d)$ were calculated from the adopted $E(B-V)$ color excesses and the apparent distance moduli listed in Table 3. The errors in the distance determinations were calculated from the expression $\sigma(d)=0.46\left\{\sigma\left(V-M_{v}\right)\right.$ $+3 \sigma[E(B-V)]\} d$, where $\sigma\left(V-M_{v}\right)$ and $\sigma[E(B-V)]$ represent the standard deviations in $V-M_{v}$ and $E(B-V)$. Table 6 summarizes the final fundamental parameters obtained for each cluster.

NGC 6253 has been studied in detail by Bragaglia et al. (1997). By comparing the observed CMDs with simulations based on stellar evolutionary models in a self-consistent way, they derived the reddening, distance, and age of the cluster: $E(B-V)=0.23-0.32, d=1.5 \mathrm{kpc}$, with metallicity approximately double the solar value, and age $\approx 3$ Gyr. All these quantities are in fairly good agreement with the values obtained in this study. Furthermore, we note that the equivalent widths of the Ca II triplet lines also suggest that NGC 6253 is slightly more metal-rich than the Sun. Pismis 18, Pismis 19, and NGC 6005 have no previous determinations of their fundamental properties. Recently, Phelps et al.
(1994) observed Pismis 19 as part of an intensive CCD photometric survey of potential old open clusters. Based on their $\delta V$ index, the distance in magnitude between the turnoff and the clump stars, they concluded that Pismis 19 is significantly younger than the Hyades. Uncertainties in the $\delta V$ method for this age range may explain this.

\section{CONCLUSIONS}

We have presented CCD BVI Johnson-Cousins photometry of stars in the fields of the open cluster candidates Pismis 18, Pismis 19, NGC 6005, and NGC 6253. The photometric data on Pismis 18 and NGC 6005 supersede previous measurements by Moffat \& Vogt $(1973,1975)$, thus allowing us to extend the corresponding main sequences down to $V \approx 19$.

Pismis 18, Pismis 19, and NGC 6005 were found to be genuine open clusters. We have also identified the contribution of different disk star populations in the fields of the latter two clusters. The quality of the observed CMDs allowed us to identify the most important features in the $\mathrm{H}-\mathrm{R}$ diagram, including the main-sequence turnoff, the red giant region, and the evolved star clump, and consequently to derive reddenings, distances, and cluster ages.

We have also presented integrated spectra for Pismis 18, Pismis 19, and NGC 6253 covering a range from 3500 to $9200 \AA$ A. From the Balmer line equivalent widths and comparison of the obtained spectra with those of template clusters, reddenings and ages were derived. Cluster metallicities have also been estimated from the equivalent widths of the Ca II triplet.

The photometric and spectroscopic results indicate that Pismis 18, Pismis 19, and NGC 6005 are intermediate-age $(t \approx 1 \mathrm{Gyr})$ open clusters, while NGC 6253 is about $5 \mathrm{Gyr}$ old. The four studied clusters are located approximately in the direction of the Galactic center between 1.5 and $2.7 \mathrm{kpc}$ from the Sun, and their metallicities range over typical values of moderately metal-poor to moderately metal-rich open clusters. These preliminary metallicity determinations do not modify the pattern of the radial abundance gradient and the age-metallicity relationship that hold for the Galactic disk (Friel \& Janes 1993; Piatti et al. 1995).

We acknowledge use of the CCD and data acquisition system at CASLEO, supported under US National Science Foundation grant AST 90-15827. We thank the staff at Las Campanas for their kind hospitality and assistance during the observing runs. Thanks are also due to two anonymous referees whose comments helped us to improve the manuscript. This work has been financially supported by the institutions CONICOR and CONICET (Argentina) and CNPq and FINEP (Brazil). We also aknowledge support from the Vitae and Antorchas Foundations. Work performed at LLNL is supported by the Department of Energy under contract W7405-ENG-48. 
Barbaro, G., \& Pigatto, L. 1984, A\&A, 136, 355

Bica, E. 1988, A\&A, 195, 76

Bica, E., \& Alloin, D. 1986a, A\&A, 162, 21 (BA86a) 1986b, A\&AS, 66, 171 (BA86b) 1987, A\&A, 186, 49 (BA87)

Bica, E., Alloin, D., \& Santos, J. F. C., Jr. 1990, A\&A, 235, 103

Bragaglia, A., Tessicini, G., Tosi, M., Marconi, G., \& Munari, U. 1997, MNRAS, 284, 477

Burki, G., \& Maeder, A. 1973, A\&A, 25, 71

Carraro, G., \& Patat, F. 1995, MNRAS, 276, 563

Dawson, D. W. 1978, AJ, 83, 1424

Friel, E. D., \& Janes, K. A. 1993, A\&A, 267, 75

Gutiérrez-Moreno, A., Moreno, H., Cortés, G., \& Wenderoth, E. 1988, PASP, 100, 973

Hawarden, T. G. 1975, MNRAS, 173, 233

Janes, K. A., Tilley, C., \& Lyngå, G. 1988, AJ, 95, 711

Kassis, M., Friel, E. D., \& Phelps, R. L. 1996, AJ, 111, 820

Kilian, J., Montenbruck, O., \& Nissen, P. E. 1994, A\&A, 284, 437

Landolt, A. U. 1992, AJ, 104, 340

Luck, R. E. 1994, ApJS, 91, 309

Mathieu, R. D. 1984, ApJ, 284, 643

Moffat, A. F. J., \& Vogt, H. 1973, A\&AS, 10, 135 1975, Ä\&AS, 20,155

Ng, Y. K., Bertelli, G., Chiosi, C., \& Bressan, A. 1996, A\&A, 310, 771

Phelps, R. L., Janes, K. A., \& Montgomery, K. A. 1994, AJ, 107, 1079

\section{REFERENCES}

Piatti, A. E., Bica, E., \& Clariá, J. J. 1998, A\&AS, 127, 423

Piatti, A. E., Clariá, J. J., \& Abadi, M. G. 1995, AJ, 110, 2813

Piatti, A. E., Clariá, J. J., \& Bica, E. 1998, ApJS, 116, 263

Rosvick, J. M. 1995, MNRAS, 277, 1379

Santos, J. F. C., Jr., \& Bica, E. 1993, MNRAS, 260, 915

Santos, J. F. C. Jr., Bica, E., Clariá, J. J., Piatti, A. E., Girardi, L. A., \& Dottori, H. 1995, MNRAS, 276, 1155

Schmidt, A. 1988, SPEED User's Manual (Santa María, Brazil: Univ. Federal Santa María)

Schmidt-Kaler, T. 1982, in Landolt-Börnstein New Series, Group 6, Vol. $2 \mathrm{~b}$, Stars and Star Clusters, ed. K. Schaifers \& H. H. Voigt (Berlin: Springer), 1

Seaton, M. J. 1979, MNRAS, 187, 73

Serote Roos, M., Boisson, C., \& Joly, M. 1996, A\&AS, 117, 93

Stetson, P. B. 1991, DAOPHOT II User's Manual (Victoria: Dominion Astrophys. Obs.)

Stone, R. P. S., \& Baldwin, J. A. 1983, MNRAS, 204, 347

Straižys, V. 1992, Multicolor Stellar Photometry (Tuscon: Pachart)

Terlevich, E. 1987, MNRAS, 224, 193

Thogersen, E. N., Friel, E. D., \& Fallon, B. V. 1993, PASP, 105, 1253

Turner, D. G. 1976, AJ, 81, 1125

Vázquez, R. A., Baume, G., Feinstein, A., \& Prado, P. 1996, A\&AS, 116, 75

Walker, A. R. 1985, MNRAS, 213, 889

Wielen, R. 1967, Veröff. Astron. Rechen-Inst. Heidelberg, No. 19 